\title{
Neurite orientation and dispersion density imaging: clinical utility, efficacy, and role in therapy
}

This article was published in the following Dove Press journal:

Reports in Medical Imaging

\section{Daichi Sone}

Integrative Brain Imaging Center, National Center of Neurology and Psychiatry, Tokyo, Japan
Correspondence: Daichi Sone National Center of Neurology and Psychiatry, 4-I-I Ogawa-Higashi, Kodaira, Tokyo 187-855I, Japan

Tel +8I 04234 I 27II I

Fax +8I 0423446745

Email daichisone@gmail.com
Abstract: In the field of diffusion magnetic resonance imaging (MRI) for neuroimaging, white matter tracts have traditionally been analyzed using diffusion tensor imaging (DTI) measures, such as fractional anisotropy. However, recent advances in diffusion MRI have provided further information on brain microstructures using multi-shell protocols of diffusion MRI. Neurite orientation dispersion and density imaging (NODDI) is one such emerging advanced diffusion MRI method that enables investigation of the neurite density and neurite orientation dispersion of brain microstructures. NODDI was developed as a practical and clinically feasible diffusion MRI technique to evaluate the microstructural complexity of dendrites and axons. This review shed light on recent studies on the use of NODDI in human brain. Indeed, a growing number of studies are using NODDI to examine neurological and psychiatric disorders, with most reporting its clinical utility. The time has thus come, for us to seriously consider the clinical use of NODDI.

Keywords: neurite orientation dispersion and density imaging, diffusion magnetic resonance imaging, brain image, neurological diseases, psychiatric disorders

\section{Introduction}

In the field of diffusion magnetic resonance imaging (MRI) for neuroimaging, white matter tracts have traditionally been analyzed using diffusion tensor imaging (DTI) measures, such as fractional anisotropy. ${ }^{1}$ However, recent advances in diffusion MRI have provided further information on brain microstructures using multi-shell protocols of diffusion MRI, including diffusion kurtosis imaging (DKI), q-space imaging (QSI), and restriction spectrum imaging (RSI). ${ }^{2-4}$ Neurite orientation dispersion and density imaging (NODDI) is one such emerging advanced diffusion MRI method that enables investigation of the neurite density (ND) and neurite orientation dispersion (OD) of brain microstructures. ${ }^{5}$ Conventional DTI is based on the assumption of Gaussian distribution of diffusion processes, which may be inappropriate for non-Gaussian diffusion in biological tissues. ${ }^{6}$ Therefore, DKI, which is a common non-Gaussian diffusion model, has been developed as the extension of DTI. ${ }^{3}$ However, parameters derived from DKI lacked structural specificity, and for this reason, NODDI was proposed to provide more specific indices of tissue microstructures. ${ }^{7}$ On the other hand, QSI can provide molecular displacement probability maps by using Fourier transformation, ${ }^{2}$ but the prolonged acquisition time is problematic in clinical use. ${ }^{8}$ RSI is a newer method than QSI or DKI, and also yields parameters of ND but not of OD. ${ }^{4}$ Thus, NODDI is a clinically feasible technique to provide tissue-specific information based on non-Gaussian 
diffusion model. Indeed, a growing number of studies are using NODDI to examine neurological and psychiatric disorders such as Alzheimer's disease and schizophrenia., ${ }^{9,10}$ In this article, I review the literature on recent applications of NODDI and discuss its potential clinical utility.

\section{Principles and validations}

NODDI was developed as a practical and clinically feasible diffusion MRI technique to evaluate the microstructural complexity of dendrites and axons. While OD, which represents the angular variation of neurites, can be estimated with a single high angular resolution diffusionweighted imaging (HARDI) shell, ND requires at least two shells (ie b-values). ${ }^{5}$ NODDI has an advantage over conventional DTI in regions with a complex microstructure and is feasible with 1.5-T MRI scanners. ${ }^{11}$ Histopathologically, Sepehrband et $\mathrm{al}^{12}$ reported good agreement between the fiber density estimated by NODDI and the values measured by microscopy. According to a more recent histopathological validation study, ${ }^{13}$ NODDI can adequately describe the overall angular structures of fiber OD but fails to consistently extract discrete measures of the numbers and orientations of fiber OD peaks. Thus, although there is still room for improvement, NODDI may become a useful and reliable method for further investigating the microstructural complexity of the brain.

\section{Alzheimer's disease}

Alzheimer's disease is the most common cause of dementia. Early detection of Alzheimer's disease is clinically important in terms of early cognitive intervention ${ }^{14,15,16}$ as well as future development of disease-modifying therapy, ${ }^{17}$ and neuroimaging plays key roles in ensuring accurate and early diagnosis, in revealing the underlying pathophysiology, and in monitoring the disease. Thus far, the use of NODDI in Alzheimer's disease has focused on young-onset Alzheimer's disease (Table 1). ${ }^{10,18}$ Using NODDI, Slattery et $\mathrm{al}^{10}$ reported an association between white matter changes and apolipoprotein E (APOE) $\varepsilon 4$ status, which is the main inherited risk factor for sporadic Alzheimer's disease. They also revealed correlations between regional white matter ND and neuropsychological batteries. Another study of young-onset Alzheimer's disease and NODDI reported a widespread reduction in cortical ND and OD, including mesial and lateral temporal lobe and precuneus. ${ }^{18}$ Moreover, $\mathrm{Fu}$ et $\mathrm{al}^{19}$ reported that ND has detected clearer differences between Alzheimer's disease and mild cognitive impairment than FA. Thus, NODDI may have better sensitivity to diagnose Alzheimer's disease than conventional DTI.

\section{Parkinson's disease}

Parkinson's disease is a common neurodegenerative movement disorder characterized by the degeneration of dopaminergic neurons in the substantia nigra, and the overall prevalence is approximately $300-500$ per 100,000 people. $^{20}$ Through the use of NODDI, Kamagata and colleagues ${ }^{21}$ first unveiled reduced ND in the substantia nigra and putamen in patients with Parkinson's disease, which correlated with disease severity. Subsequently, the same group also reported detection of gray matter abnormalities by NODDI and its diagnostic accuracy, as well as retrograde degeneration of the nigrostriatal pathway in patients with Parkinson's disease. ${ }^{22,23}$ Thus, NODDI may prove to be useful in the diagnosis and monitoring of Parkinson's disease. Especially, the potential of early detection has been suggested. ${ }^{7}$

\section{Multiple sclerosis}

Multiple sclerosis (MS) is an autoimmune disease involving damage to the myelin sheaths of the brain and spinal cord, and the global median prevalence is 33 per 100,000 people. ${ }^{24}$ In NODDI, the demyelinating lesions in white matter are detected as decreased ND (Figure 1). Schneider and colleagues $^{25}$ reported more sensitive detection of MS lesions with NODDI parameters than with conventional DTI. Similar findings were also reported for spinal lesions. ${ }^{26}$ In contrast, the cortical lesions of MS are more subtle and difficult to detect on conventional MRI. Accordingly, the finding of reduced ND even in cortical lesions ${ }^{27}$ may have further clinical implications for MS. Additionally, the further usefulness of NODDI was indicated for detecting the disease progression of MS, including the change from normalappearing white matter to lesions. ${ }^{28,29}$ The viability of a 7-T MRI scanner was also confirmed in MS. ${ }^{30}$

\section{Epilepsy}

Epilepsy is a common neurological disease marked by recurrent seizures associated with abnormal electrical activity in the brain. Patients with drug-resistant focal seizures can benefit from neurosurgical resection, and successful localization of the focus is thus a key clinical role of neuroimaging in epilepsy. For focal epilepsy, Winston et $\mathrm{al}^{31}$ reported the clinical usefulness of reduced ND in focal cortical dysplasia, which was also visible on conventional MRI. Subsequently, a significant reduction in ND was revealed in temporal lobe 


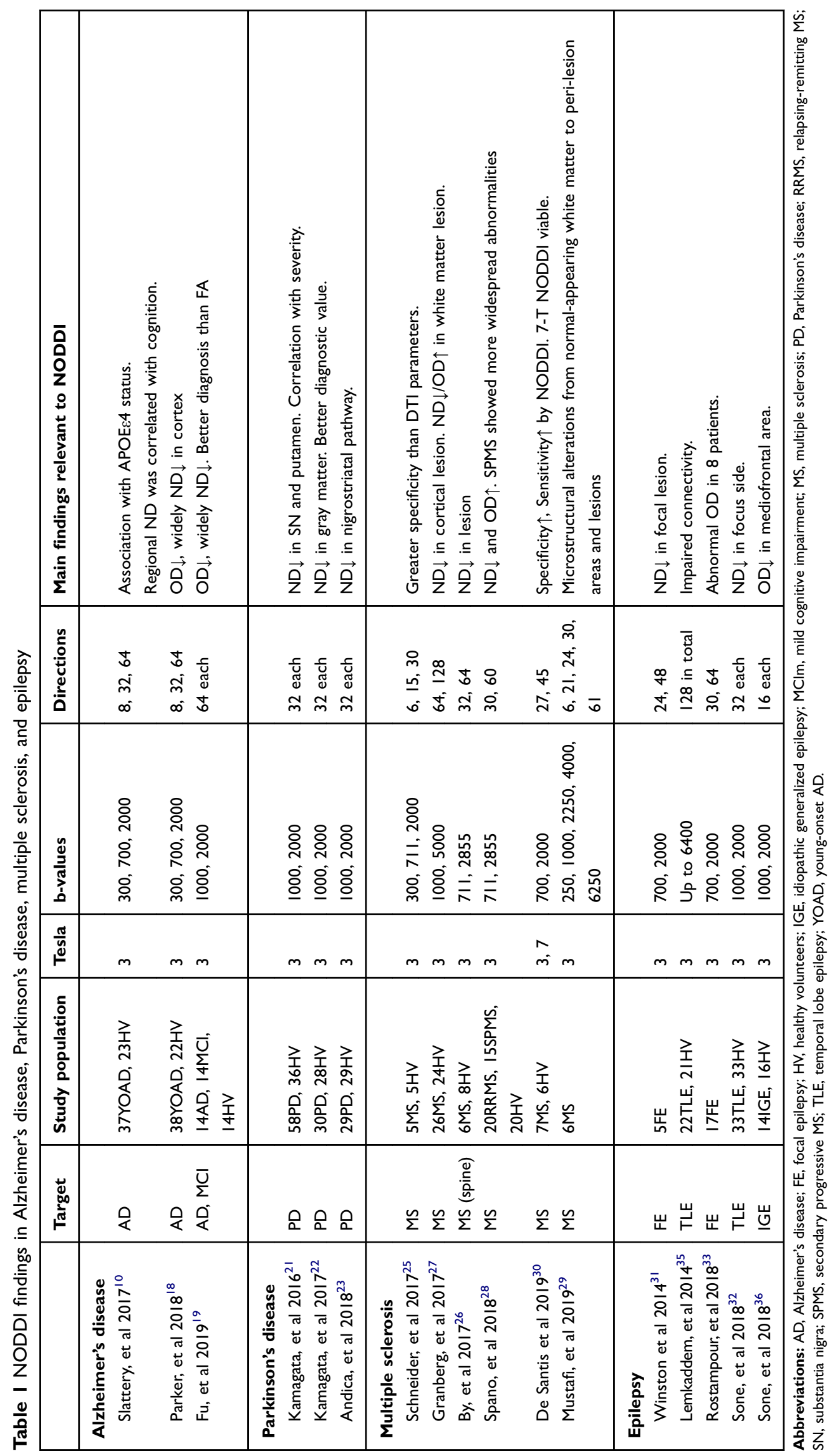




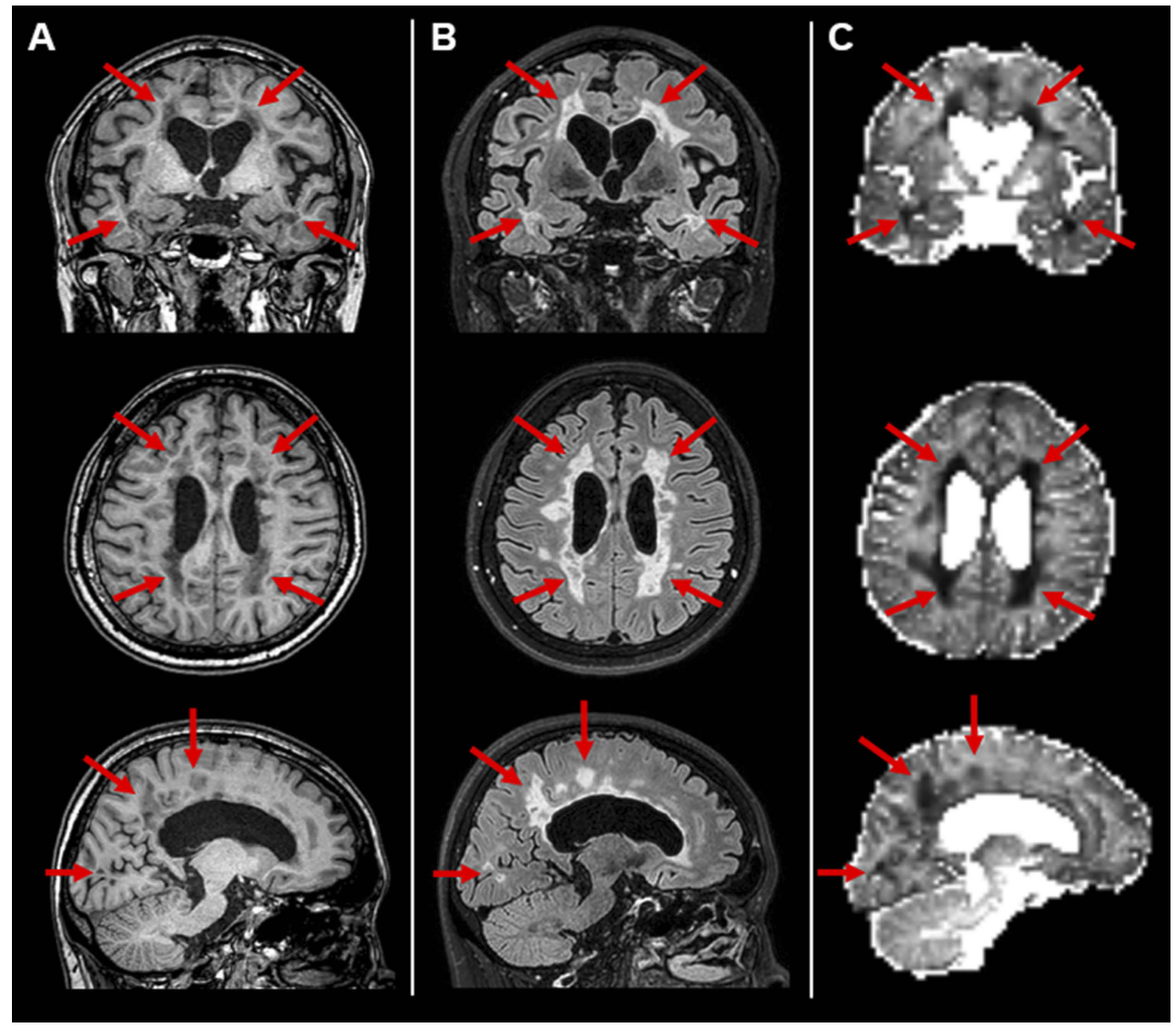

Figure I Sample images (A, TI-weighted; B, FLAIR; C, neurite density) of a 48-year-old patient with relapsing-remitting multiple sclerosis. Reduced neurite density can be seen in the demyelinating white matter lesions (arrows).

epilepsy without visible lesions on conventional MRI. ${ }^{32}$ Additionally, hippocampal sclerosis, which is the most common cause of temporal lobe epilepsy, shows reduced ND and OD. ${ }^{32}$ Representative images of these findings are shown in Figure 2. Cortical abnormalities on OD images in focal epilepsy have also been reported, ${ }^{33}$ but a limitation was raised afterward. ${ }^{34}$ At any rate, these findings would suggest a potential use for NODDI as a novel clinical biomarker for focal epilepsy treatment. Apart from focus detection, NODDI has revealed a brain connectivity dysfunction in temporal lobe epilepsy ${ }^{35}$ and a frontal lobe abnormality in idiopathic generalized epilepsy. ${ }^{36}$

\section{Stroke}

In the field of stroke, NODDI is mainly being investigated for its ability to predict and monitor recovery after stroke (Table 2). The global prevalence of stroke is approximately 500 per 100,000 people and it increases up to 4,835 in the elderly. ${ }^{37}$ To achieve a better course of treatment, prognosis of post-stroke recovery is desirable but still imprecise. ${ }^{38}$ Diffusion MRI is an expected biomarker, ${ }^{39}$ and thus NODDI may expand the predictability by providing additional information on microstructural complexity after stroke. An initial preliminary study showed greater specificity of NODDI for white matter reconstruction after brain infarction. ${ }^{40}$ Subsequently, Hodgson et $\mathrm{al}^{41}$ showed the usefulness of NODDI for predicting upper extremity motor function recovery after stroke, by investigating NODDI parameters within the corticospinal tracts. Another study revealed an increase of OD in the ipsilateral corticospinal tract in subacute stroke, which persisted in the chronic phase. ${ }^{42}$ Additionally, NODDI was reported to have improved sensitivity for detecting microstructural changes in the brain during ischemic stroke compared with DTI and DKI. ${ }^{43}$ Beyond usual stroke, Hara et $\mathrm{al}^{44}$ investigated patients with moyamoya disease, which is a progressive cerebrovascular disease caused by blocked main arteries of the brain, and found that NODDI parameters are significantly correlated with PET and clinical severity. 

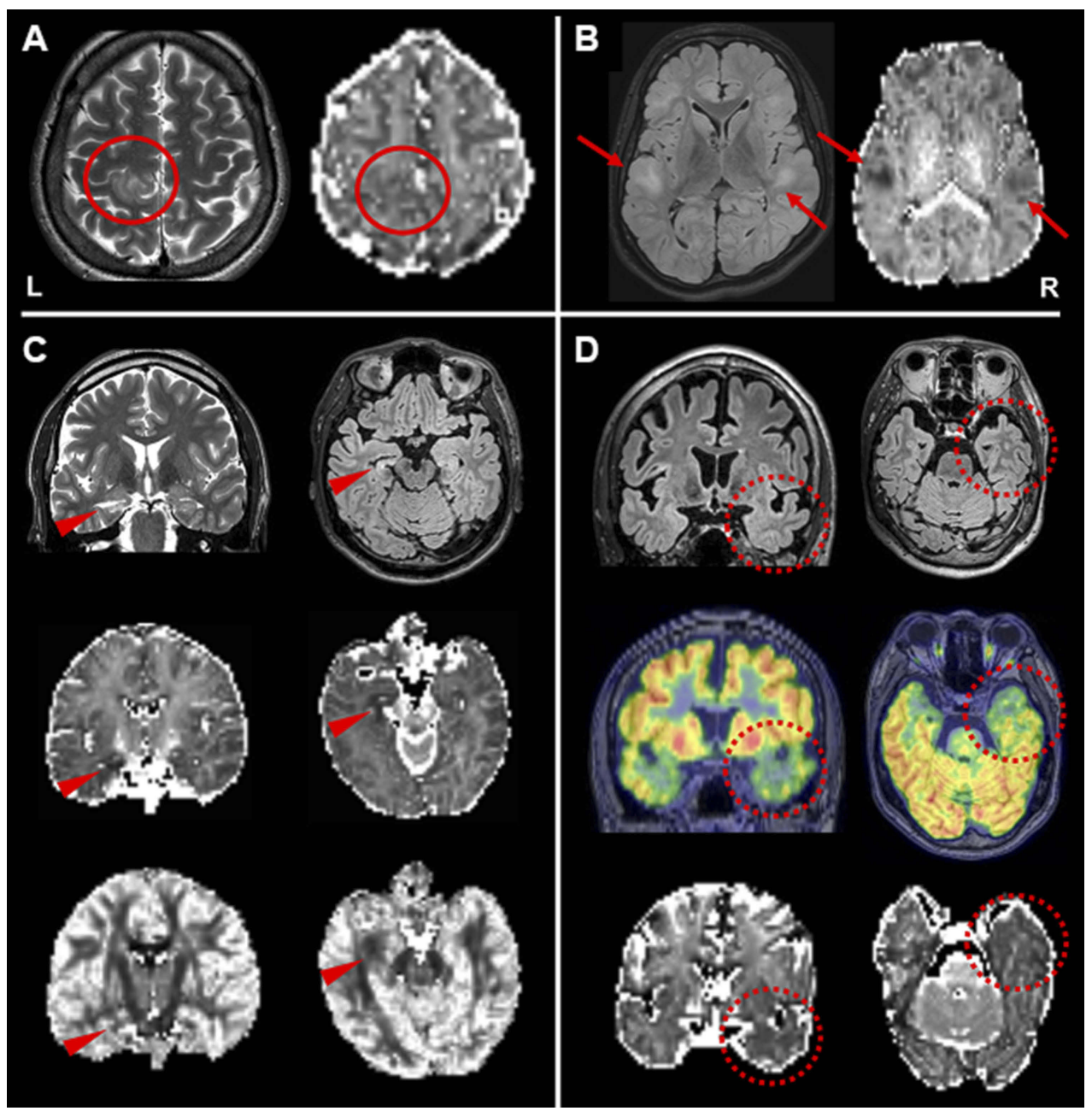

Figure 2 (A) In a 46-year-old patient with focal epilepsy, reduced neurite density is evident in the focal cortical dysplasia (circles). (B) A I7-year-old patient with tuberous sclerosis. Reduced neurite density can be seen in the multiple cortical tubers (arrows). (C) A 37-year-old patient with left temporal lobe epilepsy and hippocampal sclerosis. The abnormal hippocampus shows reduced neurite density and orientation dispersion (arrowheads). (D) A 47-year-old patient with right temporal lobe without any visible lesions on conventional MRI. The right temporal lobe shows reduced neurite density in accordance with the hypometabolic areas of ${ }^{18} \mathrm{~F}-\mathrm{FDG}-\mathrm{PET}$ (broken circles).

\section{Tumors}

The clinical application of NODDI is also expected in the field of brain tumors, particularly for differentiating gliomas. After the discovery of unique contrast of NODDI maps within gliomas with a 7-T MRI scanner, ${ }^{45}$ Maximov et $\mathrm{al}^{46}$ reported the reliable and feasible differentiation of glioma grading by NODDI parameters. Additionally, although there is no significant additional utility of NODDI for detecting isocitrate dehydrogenase-1 (IDH-1) mutation status in gliomas, ${ }^{47,48}$ quantitative NODDI metrics in tumoral and peritumoral regions are suggested to be useful for glioma grading. ${ }^{48} \mathrm{ND}$ seems to have the best discriminative power for distinguishing normal, tumoral, and peritumoral edematous areas. ${ }^{49}$ In addition, Kadota and colleagues ${ }^{50}$ showed a potential differentiation between glioblastoma and solitary metastasis with NODDI.

\section{Trauma}

For the use of NODDI in traumatic brain injury, Wu et al ${ }^{51}$ demonstrated improved sensitivity of the NODDI parameter (axonal density) for white matter changes shortly after mild traumatic brain injury. Additionally, Churchill et $\mathrm{al}^{52}$ reported a reduced ND and increased OD index in athletes with concussion. Afterward, the same group also found NODDI abnormalities at both phases in injury and recovery from concussion using longitudinal data. ${ }^{53}$ These findings may help us to understand the microstructural complexity of brain injuries over time.

\section{Psychiatric disorders}

Psychiatric disorders are also an important clinical target of advanced neuroimaging modalities. For NODDI, Nazeri and colleagues ${ }^{9}$ found significantly lower ND in gray 


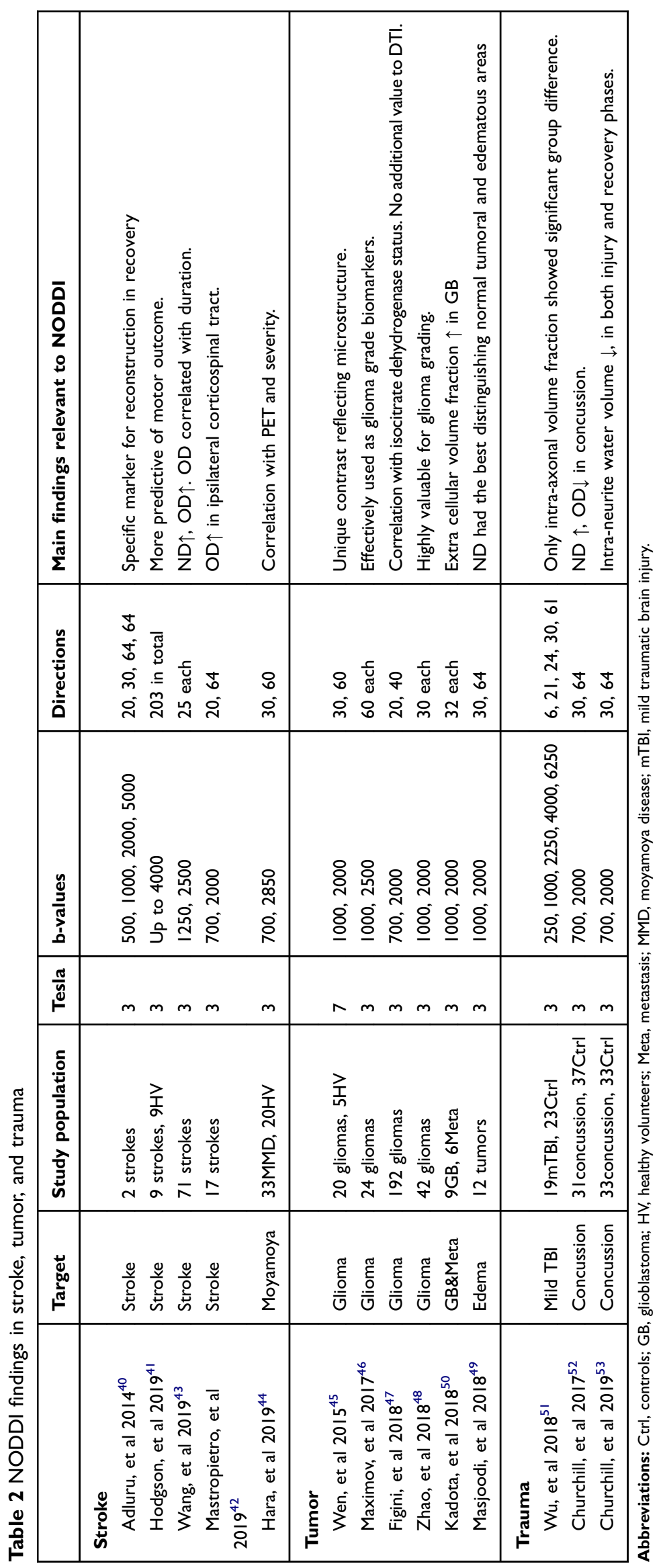


matter in the temporal pole, anterior parahippocampal gyrus, and hippocampus of patients with schizophrenia. Additionally, significantly reduced fractional anisotropy and ND in several white matter tracts were also reported in patients with first-episode psychosis. ${ }^{54}$ In schizophrenia, another study showed increased OD in the posterior limb of internal capsule and negative correlation with subsequent drug response. ${ }^{55}$ Moreover, Spray et $\mathrm{al}^{56,57}$ reported correlations between $\mathrm{OD}$ and hallucination proneness in otherwise healthy individuals. For mood disorders, reduced ND and OD were found in major depressive disorder with a correlation between OD and disease severity. ${ }^{58}$ In bipolar affective disorder, Ota et $\mathrm{al}^{59}$ reported reduced ND in the right posterior cingulate cortex, although a previous study did not find any significant changes in bipolar affective disorder. ${ }^{9}$ This discrepancy could be related with treatment, since another study reported that patient with bipolar affective disorder without lithium therapy showed lower ND in the left frontal cortex than those with lithium as well as than healthy controls. ${ }^{60}$ Thus, there are already several studies of NODDI and several common psychiatric disorders, suggesting a future clinical use for NODDI. However, psychiatric disorders and symptoms are highly diverse and remain to be fully elucidated in neuroimaging studies.

\section{Brain development}

NODDI is also suggested to be useful for estimating brain development from the neonatal period to adulthood (Table 3). In healthy infants, NODDI in combination with myelin content information may provide a good indicator of brain myelination with age. ${ }^{61}$ In addition, in preadolescence, ND is strongly correlated with age in many brain regions, showing an even better correlation than fractional anisotropy. ${ }^{62}$ Age-related increases of ND were also reported during late childhood and adolescence, suggesting axonal packing in this term. ${ }^{63}$ For adults, Nazeri and colleagues ${ }^{64}$ revealed age-related OD decreases in frontoparietal regions and increases in the hippocampus, as well as a relationship with executive function. Ota et $\mathrm{al}^{65}$ also reported age-related changes in healthy adults in terms of NODDI and DTI/DKI parameters.

As a comparison with normal development, several developmental abnormalities have been investigated by NODDI. In preterm and very preterm children, NODDI detected several regional microstructural changes, partly associated with language function or intelligence quotient. $^{66,67,68}$ Additionally, Karmacharya, et al investigated neonates with congenital heart disease and reported bilateral reduction in several tracts. ${ }^{69}$ In developmental dyslexia, age-related differences in local gyrification were correlated with NODDI parameters. ${ }^{70}$ Thus, NODDI may enable us to investigate human brain development, aging, and developmental disorders in greater detail.

\section{Other brain disorders}

There are many applications of NODDI in other brain disorders (Table 4). Broad et $\mathrm{al}^{71}$ found reduced ND in extensive regions of the corticospinal tract in amyotrophic lateral sclerosis, which is consistent with the core pathology of these areas. The corticospinal tract is also a target of research in idiopathic normal pressure hydrocephalus. ${ }^{72,73}$ In the corticospinal tract, ND and OD are reduced in idiopathic normal pressure hydrocephalus, ${ }^{72}$ and axon density was unchanged after surgical treatment. ${ }^{73}$ In preclinical Huntington's disease, widespread reductions in axonal density have already been found and correlated with clinical disease progression. ${ }^{74}$ Song et al $^{75}$ used NODDI to investigate patients with Wilson disease and found a significant reduction in ND and OD in the basal ganglia and thalamus. In galactosemia, ND was decreased in bilateral anterior areas and OD was increased in the left hemisphere. ${ }^{76}$ Another study investigating patients with diabetes mellitus and mild cognitive impairment reported reduced ND and its correlations with the hemoglobin A1C level, disease duration, and neuropsychological scores. ${ }^{77}$ The effect of systemic medical conditions on the brain was also investigated using NODDI for hypertension, ${ }^{78}$ sickle cell anemia, ${ }^{79}$ and interferon-alpha-induced fatigue. ${ }^{80}$ Abnormal NODDI parameters were also reported in patients with myalgic encephalomyelitis/chronic fatigue syndrome. ${ }^{81}$ Billiet and colleagues $^{82}$ found reduced ND in the "unidentified bright objects" in neurofibromatosis type 1. Other studies focused on perinatal encephalopathy, ${ }^{83}$ SYN1Q555X mutation, ${ }^{84}$ C9orf72 disease, ${ }^{85}$ and sarcoma survivors. ${ }^{86}$

\section{Spine}

NODDI is also feasible for evaluating the human spinal cord. ${ }^{87}$ In addition to its use for the spinal lesions of $\mathrm{MS},{ }^{26,88}$ some studies have focused on cervical spondylotic myelopathy. ${ }^{89,90,91}$ In general, ND showed good correlations with disease severity. In particular, preoperative ND may be a strong indicator of neurological dysfunction and postoperative recovery in cervical spondylotic myelopathy. $^{91}$ 


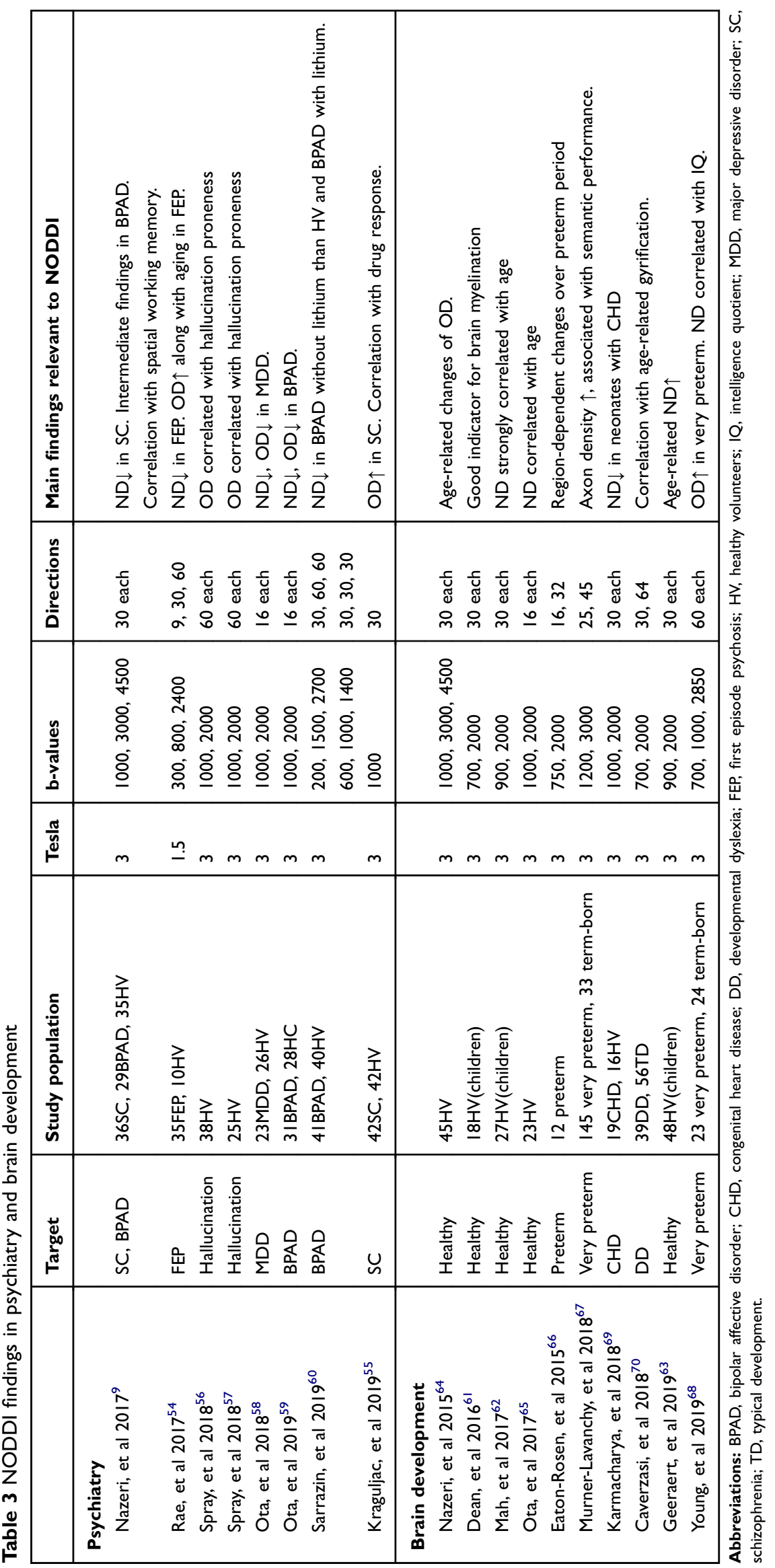




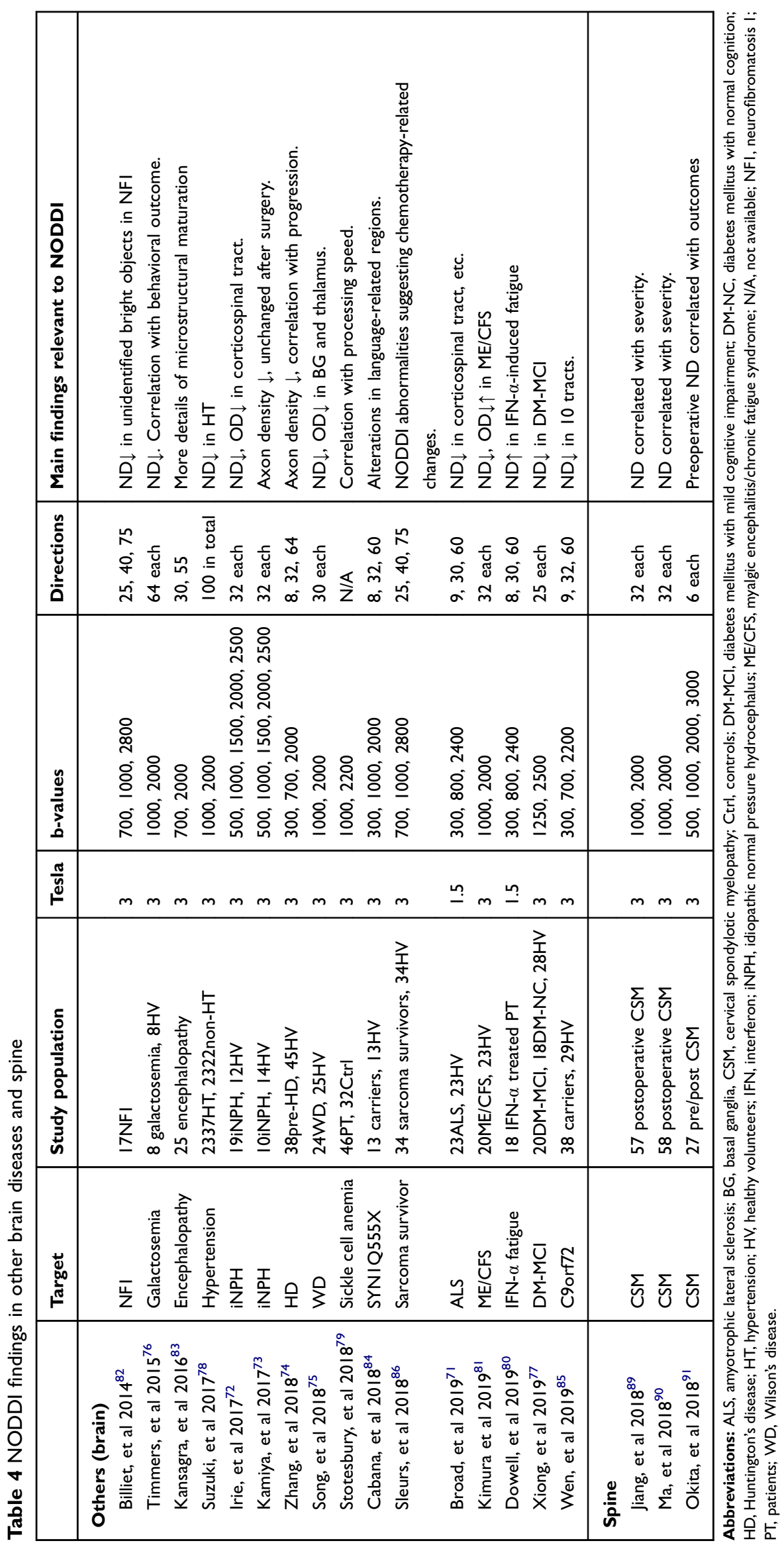




\section{Future directions}

Because the number of studies of NODDI is increasing, we should seriously consider the use of NODDI in daily clinical practice. However, there are several issues to be addressed. First, the MRI protocols and scanners are still inconsistent, and no standards have been established. To achieve the clinical utility of NODDI across different institutes, standard settings should be needed. Although optimal b-values and more directions may yield better images, a clinical protocol with a shorter acquisition time is preferable. Second, we have to consider which NODDI parameters to use. Beyond ND and OD, we can also obtain more advanced metrics such as axon density. Similarly, regarding the targeted areas of brain (eg gray matter or white matter tract), there's some variability among studies. We may need to understand the basic and clinical meanings of these parameters to correctly interpret patients' findings. Third, most of the clinical studies on NODDI are from single centers and have not been reproduced in other studies. Novelty is an important consideration for study and publication, but clinical application also strongly requires further accumulation of evidence from repeated confirmation of results.

In addition, there are some useful tools or improved methodologies. Cacerzasi et $\mathrm{al}^{92}$ reported the use of color maps of NODDI for the visual assessment of several neurological diseases. On the other hand, the criticisms to NODDI are mainly associated with the bias derived from the fitting model and its assumption..$^{93,94,95}$ To overcome this limitation, there are improved methodologies for diffusion MRI, such as NODDI with diffusivity assessment (NODDIDA), ${ }^{94}$ spherical mean technique (SMT) ${ }^{95}$ NODDI-DTI model ${ }^{96}$ and gamma metrics. ${ }^{97}$ These methodological advances may also deepen our basic and clinical knowledge and promote the clinical use of NODDI.

\section{Conclusion}

This review shed light on recent studies on the use of NODDI in humans. The number of studies using NODDI is growing, with most reporting its clinical utility. The time has thus come for us to seriously consider the clinical use of NODDI. Especially, NODDI may directly affect the treatment and recovery prediction in several diseases including stroke, epilepsy and glioma. Setting standard protocols and parameters will accelerate the clinical applications and highly benefit patients with neurological and psychiatric disorders.

\section{Acknowledgment}

I am grateful to my colleagues in the Integrative Brain Imaging Center and Department of Radiology at National Center of Neurology and Psychiatry, Japan.

\section{Disclosure}

The author reports no conflicts of interest in this work.

\section{References}

1. Kubicki M, Westin CF, Maier SE, et al. Diffusion tensor imaging and its application to neuropsychiatric disorders. Harv Rev Psychiatry. 2002;10(6):324-336.

2. Cohen Y, Assaf Y. High b-value q-space analyzed diffusion-weighted MRS and MRI in neuronal tissues - a technical review. NMR Biomed. 2002;15(7-8):516-542. doi:10.1002/nbm.778

3. Jensen JH, Helpern JA, Ramani A, Lu H, Kaczynski K. Diffusional kurtosis imaging: the quantification of non-gaussian water diffusion by means of magnetic resonance imaging. Magn Reson Med. 2005;53 (6):1432-1440. doi:10.1002/mrm.20508

4. White NS, Leergaard TB, D'Arceuil H, Bjaalie JG, Dale AM. Probing tissue microstructure with restriction spectrum imaging: histological and theoretical validation. Hum Brain Mapp. 2013;34 (2):327-346. doi:10.1002/hbm.21454

5. Zhang H, Schneider T, Wheeler-Kingshott CA, Alexander DC. NODDI: practical in vivo neurite orientation dispersion and density imaging of the human brain. Neuroimage. 2012;61(4):1000-1016. doi:10.1016/j.neuroimage.2012.03.072

6. De Santis S, Gabrielli A, Palombo M, Maraviglia B, Capuani S. Non-Gaussian diffusion imaging: a brief practical review. Magn Reson Imaging. 2011;29(10):1410-1416. doi:10.1016/j.mri.2011. 04.006

7. Kamagata K, Hatano T, Aoki S. What is NODDI and what is its role in Parkinson's assessment?. Expert Rev Neurother. 2016;16(3):241243. doi:10.1586/14737175.2016.1142876

8. Sakai K, Yamada K, Akazawa K, et al. Can we shorten the q-space imaging to make it clinically feasible? Jpn J Radiol. 2017;35(1):1624. doi:10.1007/s11604-016-0593-8

9. Nazeri A, Mulsant BH, Rajji TK, et al. Gray matter neuritic microstructure deficits in schizophrenia and bipolar disorder. Biol Psychiatry. 2017;82(10):726-736. doi:10.1016/j.biopsych.2016.12. 005

10. Slattery CF, Zhang J, Paterson RW, et al. ApoE influences regional white-matter axonal density loss in Alzheimer's disease. Neurobiol Aging. 2017;57:8-17. doi:10.1016/j.neurobiolaging.2017.04.021

11. Chung AW, Seunarine KK, Clark CA. NODDI reproducibility and variability with magnetic field strength: A comparison between $1.5 \mathrm{~T}$ and 3 T. Hum Brain Mapp. 2016;37(12):4550-4565. doi:10.1002/ hbm. 23328

12. Sepehrband F, Clark KA, Ullmann JF, et al. Brain tissue compartment density estimated using diffusion-weighted MRI yields tissue parameters consistent with histology. Hum Brain Mapp. 2015;36 (9):3687-3702. doi:10.1002/hbm.22872

13. Schilling KG, Janve V, Gao Y, Stepniewska I, Landman BA, Anderson AW. Histological validation of diffusion MRI fiber orientation distributions and dispersion. Neuroimage. 2018;165:200-221. doi:10.1016/j.neuroimage.2017.10.046

14. Hill NT, Mowszowski L, Naismith SL, Chadwick VL, Valenzuela M, Lampit A. Computerized cognitive training in older adults with mild cognitive impairment or dementia: a systematic review and metaanalysis. Am J Psychiatry. 2017;174(4):329-340. doi:10.1176/appi. ajp. 2016.16030360 
15. Sherman DS, Mauser J, Nuno M, Sherzai D. The efficacy of cognitive intervention in Mild Cognitive Impairment (MCI): a meta-analysis of outcomes on neuropsychological measures. Neuropsychol Rev. 2017;27(4):440-484. doi:10.1007/s11065-017-9363-3

16. Nousia A, Siokas V, Aretouli E, et al. Beneficial effect of multidomain cognitive training on the neuropsychological performance of patients with early-stage alzheimer's disease. Neural Plast. 2018;2018:2845176. doi:10.1155/2018/2845176

17. Counts SE, Ikonomovic MD, Mercado N, Vega IE, Mufson EJ. Biomarkers for the early detection and progression of Alzheimer's Disease. Neurotherapeutics. 2017;14(1):35-53. doi:10.1007/s13311016-0481-z

18. Parker TD, Slattery CF, Zhang J, et al. Cortical microstructure in young onset alzheimer's disease using neurite orientation dispersion and density imaging. Hum Brain Mapp. 2018;39(7):3005-3017. doi:10.1002/hbm. 24056

19. Fu X, Shrestha S, Sun M, et al. Microstructural white matter alterations in mild cognitive impairment and alzheimer's disease: study based on Neurite Orientation Dispersion and Density Imaging (NODDI). Clin Neuroradiol. 2019. doi:10.1007/s00062-019-00805-0

20. Pringsheim T, Jette N, Frolkis A, Steeves TD. The prevalence of parkinson's disease: a systematic review and meta-analysis. Mov Disord. 2014;29(13):1583-1590. doi:10.1002/mds.25945

21. Kamagata K, Hatano T, Okuzumi A, et al. Neurite orientation dispersion and density imaging in the substantia nigra in idiopathic Parkinson disease. Eur Radiol. 2016;26(8):2567-2577. doi:10.1007/ s00330-015-4066-8

22. Kamagata K, Zalesky A, Hatano T, et al. Gray matter abnormalities in idiopathic parkinson's disease: evaluation by diffusional kurtosis imaging and neurite orientation dispersion and density imaging. Hum Brain Mapp. 2017. doi:10.1002/hbm.23628

23. Andica C, Kamagata K, Hatano T, et al. Neurite orientation dispersion and density imaging of the nigrostriatal pathway in Parkinson's disease: retrograde degeneration observed by tract-profile analysis. Parkinsonism Relat Disord. 2018;51:55-60. doi:10.1016/j. parkreldis.2018.02.046

24. Oh J, Vidal-Jordana A, Montalban X. Multiple sclerosis: clinical aspects. Curr Opin Neurol. 2018;31(6):752-759. doi:10.1097/ WCO.0000000000000622

25. Schneider T, Brownlee W, Zhang H, Ciccarelli O, Miller DH, Wheeler-Kingshott CG. Sensitivity of multi-shell NODDI to multiple sclerosis white matter changes: a pilot study. Funct Neurol. 2017;32 (2):97-101.

26. By S, Xu J, Box BA, Bagnato FR, Smith SA. Application and evaluation of NODDI in the cervical spinal cord of multiple sclerosis patients. Neuroimage Clin. 2017;15:333-342. doi:10.1016/j.nicl. 2017.05.010

27. Granberg T, Fan Q, Treaba CA, et al. In vivo characterization of cortical and white matter neuroaxonal pathology in early multiple sclerosis. Brain. 2017;140(11):2912-2926. doi:10.1093/brain/awx247

28. Spano B, Giulietti G, Pisani V, et al. Disruption of neurite morphology parallels MS progression. Neurol Neuroimmunol Neuroinflamm. 2018;5(6):e502. doi:10.1212/NXI.0000000000000502

29. Mustafi SM, Harezlak J, Kodiweera C, et al. Detecting white matter alterations in multiple sclerosis using advanced diffusion magnetic resonance imaging. Neural Regen Res. 2019;14(1):114-123. doi:10.4103/1673-5374.243716

30. De Santis S, Bastiani M, Droby A, et al. Characterizing microstructural tissue properties in multiple sclerosis with diffusion MRI at 7T and 3T: the impact of the experimental design. Neuroscience. 2019;403:17-26. doi:10.1016/j.neuroscience.2018.03.048

31. Winston GP, Micallef C, Symms MR, Alexander DC, Duncan JS, Zhang H. Advanced diffusion imaging sequences could aid assessing patients with focal cortical dysplasia and epilepsy. Epilepsy Res. 2014;108(2):336-339. doi:10.1016/j.eplepsyres.2013.11.004
32. Sone D, Sato N, Ota M, Maikusa N, Kimura Y, Matsuda H. Abnormal neurite density and orientation dispersion in unilateral temporal lobe epilepsy detected by advanced diffusion imaging. Neuroimage Clin. 2018;20:772-782. doi:10.1016/j.nicl.2018.09.017

33. Rostampour M, Hashemi H, Najibi SM, Oghabian MA. Detection of structural abnormalities of cortical and subcortical gray matter in patients with MRI-negative refractory epilepsy using neurite orientation dispersion and density imaging. Phys Med. 2018;48:47-54. doi:10.1016/j.ejmp.2018.03.005

34. Chougar L, Hagiwara A, Maekawa T, et al. Limitation of neurite orientation dispersion and density imaging for the detection of focal cortical dysplasia with a "transmantle sign". Phys Med. 2018;52:183184. doi:10.1016/j.ejmp.2018.06.011

35. Lemkaddem A, Daducci A, Kunz N, et al. Connectivity and tissue microstructural alterations in right and left temporal lobe epilepsy revealed by diffusion spectrum imaging. Neuroimage Clin. 2014;5:349-358. doi:10.1016/j.nicl.2014.07.013

36. Sone D, Watanabe M, Ota M, et al. Subtle abnormality in neurite dispersion in idiopathic generalized epilepsy detected by an advanced diffusion imaging technique. Epilepsy Seizure. 2018;10(1):33-43. doi: $10.3805 /$ eands. 10.33

37. Feigin VL, Forouzanfar MH, Krishnamurthi R, et al. Global and regional burden of stroke during 1990-2010: findings from the global burden of disease study 2010. Lancet. 2014;383(9913):245-254. doi:10.1016/s0140-6736(13)61953-4

38. Jampathong N, Laopaiboon M, Rattanakanokchai S, Pattanittum P. Prognostic models for complete recovery in ischemic stroke: a systematic review and meta-analysis. BMC Neurol. 2018;18(1):26. doi:10.1186/s12883-018-1032-5

39. Boyd LA, Hayward KS, Ward NS, et al. Biomarkers of stroke recovery: consensus-based core recommendations from the stroke recovery and rehabilitation roundtable. Neurorehabil Neural Repair. 2017;31(10-11):864-876. doi:10.1177/1545968317732680

40. Adluru G, Gur Y, Anderson JS, Richards LG, Adluru N, DiBella EV. Assessment of white matter microstructure in stroke patients using NODDI. Conf Proc IEEE Eng Med Biol Soc. 2014;2014:742-745. doi:10.1109/EMBC.2014.6943697

41. Hodgson K, Adluru G, Richards LG, et al. Predicting motor outcomes in stroke patients using diffusion spectrum mri microstructural measures. Front Neurol. 2019;10:72. doi:10.3389/fneur.2019.00072

42. Mastropietro A, Rizzo G, Fontana L, et al. Microstructural characterization of corticospinal tract in subacute and chronic stroke patients with distal lesions by means of advanced diffusion MRI. Neuroradiology. 2019. doi:10.1007/s00234-019-02249-2

43. Wang Z, Zhang S, Liu C, et al. A study of neurite orientation dispersion and density imaging in ischemic stroke. Magn Reson Imaging. 2019;57:28-33. doi:10.1016/j.mri.2018.10.018

44. Hara S, Hori M, Ueda R, et al. Unraveling specific brain microstructural damage in moyamoya disease using diffusion magnetic resonance imaging and positron emission tomography. $J$ Stroke Cerebrovasc Dis. 2019;28(4):1113-1125. doi:10.1016/j.jstrokecere brovasdis.2018.12.038

45. Wen Q, Kelley DA, Banerjee S, et al. Clinically feasible NODDI characterization of glioma using multiband EPI at 7 T. Neuroimage Clin. 2015;9:291-299. doi:10.1016/j.nicl.2015.08.017

46. Maximov II, Tonoyan AS, Pronin IN. Differentiation of glioma malignancy grade using diffusion MRI. Phys Med. 2017;40:24-32. doi:10.1016/j.ejmp.2017.07.002

47. Figini M, Riva M, Graham M, et al. Prediction of isocitrate dehydrogenase genotype in brain gliomas with mri: single-shell versus multishell diffusion models. Radiology. 2018;289(3):788-796. doi:10.1148/radiol.2018180054

48. Zhao J, Li JB, Wang JY, et al. Quantitative analysis of neurite orientation dispersion and density imaging in grading gliomas and detecting IDH-1 gene mutation status. Neuroimage Clin. 2018;19:174-181. doi:10.1016/j.nicl.2018.04.011 
49. Masjoodi S, Hashemi H, Oghabian MA, Sharifi G. Differentiation of edematous, tumoral and normal areas of brain using diffusion tensor and neurite orientation dispersion and density imaging. $J$ Biomed Phys Eng. 2018;8(3):251-260.

50. Kadota Y, Hirai T, Azuma M, et al. Differentiation between glioblastoma and solitary brain metastasis using neurite orientation dispersion and density imaging. $J$ Neuroradiol. 2018. doi:10.1016/j. neurad.2018.10.005

51. Wu YC, Mustafi SM, Harezlak J, Kodiweera C, Flashman LA, McAllister TW. Hybrid diffusion imaging in mild traumatic brain injury. $J$ Neurotrauma. 2018;35(20):2377-2390. doi:10.1089/neu.2017.5566

52. Churchill NW, Caverzasi E, Graham SJ, Hutchison MG, Schweizer TA. White matter microstructure in athletes with a history of concussion: comparing diffusion tensor imaging (DTI) and neurite orientation dispersion and density imaging (NODDI). Hum Brain Mapp. 2017;38(8):4201-4211. doi:10.1002/hbm.23658

53. Churchill NW, Caverzasi E, Graham SJ, Hutchison MG, Schweizer TA. White matter during concussion recovery: comparing diffusion tensor imaging (DTI) and neurite orientation dispersion and density imaging (NODDI). Hum Brain Mapp. 2019;40(6):1908-1918. doi: $10.1002 / \mathrm{hbm} .24500$

54. Rae CL, Davies G, Garfinkel SN, et al. Deficits in neurite density underlie white matter structure abnormalities in first-episode psychosis. Biol Psychiatry. 2017;82(10):716-725. doi:10.1016/j.biopsych. 2017.02.008

55. Kraguljac NV, Anthony T, Monroe WS, et al. A longitudinal neurite and free water imaging study in patients with a schizophrenia spectrum disorder. Neuropsychopharmacology. 2019. doi:10.1038/ s41386-019-0427-3

56. Spray A, Beer AL, Bentall RP, Sluming V, Meyer G. Relationship between hallucination proneness and musical aptitude is mediated by microstructure in the corpus callosum. Schizophr Res. 2018;197:579580. doi:10.1016/j.schres.2017.11.024

57. Spray A, Beer AL, Bentall RP, Sluming V, Meyer G. Microstructure of the superior temporal gyrus and hallucination proneness - a multicompartment diffusion imaging study. Neuroimage Clin. 2018;20:16. doi:10.1016/j.nicl.2018.06.027

58. Ota M, Noda T, Sato N, et al. The use of diffusional kurtosis imaging and neurite orientation dispersion and density imaging of the brain in major depressive disorder. $J$ Psychiatr Res. 2018;98:22-29. doi:10.1016/j.jpsychires.2017.12.011

59. Ota M, Noda T, Sato N, et al. The use of diffusional kurtosis imaging and neurite orientation dispersion and density imaging of the brain in bipolar disorder. $J$ Affect Disord. 2019;251:231-234. doi:10.1016/j. jad.2019.03.068

60. Sarrazin S, Poupon C, Teillac A, et al. Higher in vivo cortical intracellular volume fraction associated with lithium therapy in bipolar disorder: a multicenter NODDI study. Psychother Psychosom. 2019;88(3):171-176. doi:10.1159/000498854

61. Dean DC 3rd, O'Muircheartaigh J, Dirks H, et al. Mapping an index of the myelin g-ratio in infants using magnetic resonance imaging. Neuroimage. 2016;132:225-237. doi:10.1016/j.neuroimage.2016.02.040

62. Mah A, Geeraert B, Lebel C. Detailing neuroanatomical development in late childhood and early adolescence using NODDI. PLoS One. 2017;12(8):e0182340. doi:10.1371/journal.pone.0182340

63. Geeraert BL, Lebel RM, Lebel C. A multiparametric analysis of white matter maturation during late childhood and adolescence. Hum Brain Mapp. 2019. doi:10.1002/hbm.24706

64. Nazeri A, Chakravarty MM, Rotenberg DJ, et al. Functional consequences of neurite orientation dispersion and density in humans across the adult lifespan. $J$ Neurosci. 2015;35(4):1753-1762. doi:10.1523/JNEUROSCI.3979-14.2015

65. Ota M, Sato N, Maikusa N, Sone D, Matsuda H, Kunugi H. Whole brain analyses of age-related microstructural changes quantified using different diffusional magnetic resonance imaging methods. Jpn $J$ Radiol. 2017;35(10):584-589. doi:10.1007/s11604-017-0670-7
66. Eaton-Rosen Z, Melbourne A, Orasanu E, et al. Longitudinal measurement of the developing grey matter in preterm subjects using multi-modal MRI. Neuroimage. 2015;111:580-589. doi:10.1016/j. neuroimage.2015.02.010

67. Murner-Lavanchy IM, Kelly CE, Reidy N, et al. White matter microstructure is associated with language in children born very preterm. Neuroimage Clin. 2018;20:808-822. doi:10.1016/j.nicl.2018.09.020

68. Young JM, Vandewouw MM, Mossad SI, et al. White matter microstructural differences identified using multi-shell diffusion imaging in six-year-old children born very preterm. Neuroimage Clin. 2019;23:101855. doi:10.1016/j.nicl.2019.101855

69. Karmacharya S, Gagoski B, Ning L, et al. Advanced diffusion imaging for assessing normal white matter development in neonates and characterizing aberrant development in congenital heart disease. Neuroimage Clin. 2018;19:360-373. doi:10.1016/j.nicl.2018.04.032

70. Caverzasi E, Mandelli ML, Hoeft F, et al. Abnormal age-related cortical folding and neurite morphology in children with developmental dyslexia. Neuroimage Clin. 2018;18:814-821. doi:10.1016/j. nicl.2018.03.012

71. Broad RJ, Gabel MC, Dowell NG, et al. Neurite orientation and dispersion density imaging (NODDI) detects cortical and corticospinal tract degeneration in ALS. J Neurol Neurosurg Psychiatry. 2019;90(4):404-411. doi:10.1136/jnnp-2018-318830

72. Irie R, Tsuruta K, Hori M, et al. Neurite orientation dispersion and density imaging for evaluation of corticospinal tract in idiopathic normal pressure hydrocephalus. Jpn J Radiol. 2017;35(1):25-30. doi:10.1007/s11604-016-0594-7

73. Kamiya K, Hori M, Irie R, et al. Diffusion imaging of reversible and irreversible microstructural changes within the corticospinal tract in idiopathic normal pressure hydrocephalus. Neuroimage Clin. 2017;14:663-671. doi:10.1016/j.nicl.2017.03.003

74. Zhang J, Gregory S, Scahill RI, et al. In vivo characterization of white matter pathology in premanifest huntington's disease. Ann Neurol. 2018;84(4):497-504. doi:10.1002/ana.25309

75. Song YK, Li XB, Huang XL, et al. A study of neurite orientation dispersion and density imaging in wilson's disease. J Magn Reson Imaging. 2018;48(2):423-430. doi:10.1002/jmri.25930

76. Timmers I, Zhang H, Bastiani M, Jansma BM, Roebroeck A, RubioGozalbo ME. White matter microstructure pathology in classic galactosemia revealed by neurite orientation dispersion and density imaging. J Inherit Metab Dis. 2015;38(2):295-304. doi:10.1007/ s10545-014-9780-x

77. Xiong Y, Zhang S, Shi J, Fan Y, Zhang Q, Zhu W. Application of neurite orientation dispersion and density imaging to characterize brain microstructural abnormalities in type-2 diabetics with mild cognitive impairment. J Magn Reson Imaging. 2019. doi:10.1002/ jmri.26687

78. Suzuki H, Gao H, Bai W, et al. Abnormal brain white matter microstructure is associated with both pre-hypertension and hypertension. PLoS One. 2017;12(11):e0187600. doi:10.1371/journal.pone.0187600

79. Stotesbury H, Kirkham FJ, Kolbel M, et al. White matter integrity and processing speed in sickle cell anemia. Neurology. 2018;90(23): e2042-e2050. doi:10.1212/WNL.0000000000005644

80. Dowell NG, Bouyagoub S, Tibble J, Voon V, Cercignani M, Harrison NA. Interferon-alpha-induced changes in NODDI predispose to the development of fatigue. Neuroscience. 2019;403:111-117. doi:10.10 16/j.neuroscience.2017.12.040

81. Kimura Y, Sato N, Ota M, et al. Brain abnormalities in myalgic encephalomyelitis/chronic fatigue syndrome: evaluation by diffusional kurtosis imaging and neurite orientation dispersion and density imaging. $J$ Magn Reson Imaging. 2019;49(3):818-824. doi:10.1002/jmri.26247

82. Billiet T, Madler B, D'Arco F, et al. Characterizing the microstructural basis of "unidentified bright objects" in neurofibromatosis type 1: A combined in vivo multicomponent $\mathrm{T} 2$ relaxation and multi-shell diffusion MRI analysis. Neuroimage Clin. 2014;4:649-658. doi:10.10 16/j.nicl.2014.04.005 
83. Kansagra AP, Mabray MC, Ferriero DM, Barkovich AJ, Xu D, Hess CP. Microstructural maturation of white matter tracts in encephalopathic neonates. Clin Imaging. 2016;40(5):1009-1013. doi:10.1016/j. clinimag.2016.05.009

84. Cabana JF, Gilbert G, Letourneau-Guillon L, et al. Effects of SYN1Q555X mutation on cortical gray matter microstructure. Hum Brain Mapp. 2018;39(8):3428-3448. doi:10.1002/hbm.24186

85. Wen J, Zhang H, Alexander DC, et al. Neurite density is reduced in the presymptomatic phase of C9orf72 disease. J Neurol Neurosurg Psychiatry. 2019;90(4):387-394. doi:10.1136/jnnp-2018-318994

86. Sleurs C, Lemiere J, Christiaens D, et al. Advanced MR diffusion imaging and chemotherapy-related changes in cerebral white matter microstructure of survivors of childhood bone and soft tissue sarcoma? Hum Brain Mapp. 2018;39(8):3375-3387. doi:10.1002/ hbm. 24082

87. Grussu F, Schneider T, Zhang H, Alexander DC, Wheeler-Kingshott CA. Neurite orientation dispersion and density imaging of the healthy cervical spinal cord in vivo. Neuroimage. 2015;111:590-601. doi:10.1016/j.neuroimage.2015.01.045

88. Grussu F, Schneider T, Tur C, et al. Neurite dispersion: a new marker of multiple sclerosis spinal cord pathology? Ann Clin Transl Neurol. 2017;4(9):663-679. doi:10.1002/acn3.445

89. Jiang W, Han X, Guo H, et al. Usefulness of conventional magnetic resonance imaging, diffusion tensor imaging and neurite orientation dispersion and density imaging in evaluating postoperative function in patients with cervical spondylotic myelopathy. J Orthop Translat. 2018;15:59-69. doi:10.1016/j.jot.2018.08.006

90. Ma X, Han X, Jiang W, et al. A follow-up study of postoperative DCM patients using diffusion MRI with DTI and NODDI. Spine (Phila Pa 1976). 2018;43(15):E898-E904. doi:10.1097/BRS.0000 000000002541
91. Okita G, Ohba T, Takamura T, et al. Application of neurite orientation dispersion and density imaging or diffusion tensor imaging to quantify the severity of cervical spondylotic myelopathy and to assess postoperative neurologic recovery. Spine J. 2018;18(2):268275. doi:10.1016/j.spinee.2017.07.007

92. Caverzasi E, Papinutto N, Castellano A, et al. Neurite orientation dispersion and density imaging color maps to characterize brain diffusion in neurologic disorders. $J$ Neuroimaging. 2016;26(5):494498. doi:10.1111/jon. 12359

93. Jelescu IO, Veraart J, Adisetiyo V, Milla SS, Novikov DS, Fieremans E. One diffusion acquisition and different white matter models: how does microstructure change in human early development based on WMTI and NODDI? Neuroimage. 2015;107:242-256. doi:10.1016/j. neuroimage.2014.12.009

94. Jelescu IO, Veraart J, Fieremans E, Novikov DS. Degeneracy in model parameter estimation for multi-compartmental diffusion in neuronal tissue. NMR Biomed. 2016;29(1):33-47. doi:10.1002/ nbm. 3450

95. Kaden E, Kelm ND, Carson RP, Does MD, Alexander DC. Multicompartment microscopic diffusion imaging. Neuroimage. 2016;139:346-359. doi:10.1016/j.neuroimage.2016.06.002

96. Edwards LJ, Pine KJ, Ellerbrock I, Weiskopf N, Mohammadi S. NODDI-DTI: estimating neurite orientation and dispersion parameters from a diffusion tensor in healthy white matter. Front Neurosci. 2017;11:720. doi:10.3389/fnins.2017.00720

97. Guerreri M, Palombo M, Caporale A, et al. Age-related microstructural and physiological changes in normal brain measured by MRI gamma-metrics derived from anomalous diffusion signal representation. Neuroimage. 2019;188:654-667. doi:10.1016/j.neuroimage. 2018.12.044

\section{Publish your work in this journal}

Reports in Medical Imaging is an international, peer-reviewed, open access journal publishing original research, reports, reviews and commentaries on all areas of medical imaging. The manuscript management system is completely online and includes a very quick and fair peerreview system, which is all easy to use. Visit http://www.dovepress. com/testimonials.php to read real quotes from published authors. 